\title{
Complex TNF- $\alpha$ B cell epitope MAP vaccine alleviates murine ulcerative colitis
}

\author{
YAN SUN ${ }^{1}$, WENSHENG PAN ${ }^{2,3}$, JUN ZHANG $^{2,3}$, YING CUI $^{4}$, HUIJU WANG $^{5}$, GUOQING RU $^{6}$ and LEGAO CHEN ${ }^{7}$ \\ ${ }^{1}$ The Second Clinical Medical College, Zhejiang Chinese Medical University, Hangzhou, Zhejiang 310053; \\ ${ }^{2}$ Department of Gastroenterology, People's Hospital of Hangzhou Medical College; ${ }^{3}$ Department of Gastroenterology, \\ Zhejiang Provincial People's Hospital; ${ }^{4}$ Department of Nuclear Medicine, ${ }^{5}$ Key Laboratory of \\ Gastroenterology of Zhejiang Province, Departments of ${ }^{6}$ Pathology and ${ }^{7}$ Vascular Surgery, People's Hospital of \\ Hangzhou Medical College, Hangzhou, Zhejiang 310014, P.R. China
}

Received November 25, 2018; Accepted July 1, 2019

DOI: $10.3892 /$ ijmm.2019.4271

\begin{abstract}
The present study aimed to develop a tumor necrosis factor- $\alpha$ (TNF- $\alpha$ ) B-cell epitope/IL-1 $\beta$ helper T lymphocyte epitope complex MAP vaccine for the alleviation of ulcerative colitis (UC) in mice. The B cell epitopes of murine TNF- $\alpha$ $(\mathrm{mTNF}-\alpha)$ were predicted in silico and coupled with the universal interleukin $1 \beta$ (IL-1 $\beta$ ) helper T-cell epitope peptide VQGEESNDK to synthesize the eight-branched MAP vaccine. Then, the immunological effects of the MAP vaccine were assessed in vitro and in vivo, as well as its impacts on DAI index, serum DAO levels, colon tissue tight junction protein amounts, ultrastructural changes, and MPO activity in BALB/c mice with UC. The amino acids LTLRSSSQNSSDKPV at positions 78-92 of mTNF- $\alpha$ may constitute the dominant B cell epitope. Based on this finding, an eight-branched peptide structure, the TNF- $\alpha$ B-cell epitope/IL-1 $\beta$ helper T-cell epitope complex MAP vaccine, was synthesized. Indirect ELISA confirmed that MAP had a high affinity with commercialized mTNF- $\alpha$ antibodies. Meanwhile, MAP induced high specific antibody titers in vivo, reduced the DAI score, serum MPO activity, colorectal lymph node colony count, ultrastructural injuries, colon tissue histological index score and MPO activity in UC mice, while increasing the expression levels of occludin, claudin1 and ZO1 in colon tissues. The synthetic complex MAP vaccine has good antigenicity and immunogenicity, and can alleviate UC in mouse models.
\end{abstract}

Correspondence to: Dr Jun Zhang, Department of Gastroenterology, People's Hospital of Hangzhou Medical College, Zhejiang Provincial People's Hospital, 158 Shangtang Road, Hangzhou, Zhejiang 310014, P.R. China

E-mail: 19587372@qq.com

Key words: tumor necrosis factor- $\alpha$, epitope, multiple antigenic polypeptide, immunogenicity, ulcerative colitis

\section{Introduction}

Tumor necrosis factor- $\alpha$ (TNF- $\alpha)$ is a cytokine with complex biological activity and an autoantigen that can be used as a therapeutic target $(1,2)$. In addition to killing tumor cells in vitro and in vivo, TNF- $\alpha$ induces inflammatory responses, exhibits anti-viral effects, regulates the immune system, and promotes cell proliferation and activation (3). However, when TNF- $\alpha$ is overexpressed, it and other inflammatory factors can lead to a variety of pathological conditions, such as inflammatory bowel disease, infectious diseases and cachexia (4-6). Therefore, neutralizing excessive TNF- $\alpha$ expression in the body provides an effective tool for the treatment of such diseases (4-6). Currently, several drugs are used clinically to treat diseases by neutralizing excess TNF- $\alpha$, including a TNF- $\alpha$ human-murine chimeric antibody (infliximab), a TNF- $\alpha$ soluble receptor (etanercept), a human monoclonal antibody (aladalimumab) and a recombinant humanized anti-TNF- $\alpha$ fragment antibody (certolizumab) (5). Among these, infliximab is the most commonly used exogenous antibody, and has been approved for the clinical treatment of Crohn's disease. Clinical trials have demonstrated that infliximab exhibits positive effects in ulcerative colitis (UC), such as lowering the use of corticosteroid and reducing the colectomy rate in acute severe $\mathrm{UC}(7,8)$. However, there are certain issues involved with the aforementioned drugs, including high dosage requirements, long-term repeated use, the ability to easily induce hypersensitivity, and high costs, which hinder their widespread use (9). In addition, regarding the shortcomings of such monoclonal antibodies, the journal Nature published an article entitled 'Can super-antibody drugs be tamed?', which reported that the potential harm of drug-induced immune response has become a key factor limiting their use (10).

Currently, developing immunization vaccines against human self-proteins (i.e., autologous vaccines) has become a novel direction for anti-molecular immunotherapy rather than the use of monoclonal antibody drugs (11). As autologous molecules are autoantigens with small molecular weights and monomeric structures, often resulting in reduced immunogenicity; they cannot induce ideal immune responses in vivo, particularly humoral immune responses (12). Insertion of the 
exogenous universal helper T-cell epitope into the proper position of a self-antigen can help break the immune restriction and induce neutralizing antibodies against the self-molecule (13).

The newly proposed multiple antigenic polypeptide (MAP) design uses lysine as the core matrix, which has a low molecular weight and weak immunogenicity; it combines with a number of (usually 4 or 8) antigen epitopes of the same or different monomer peptides to form a dendritic structure. This pattern mimics the natural epitope conformation and can also activate humoral immunity to induce high titer and affinity antibodies without re-coupling the carrier protein (14). Based on previous investigations associated with MAP vaccines, the authors of the current study successfully enhanced heparinase immunogenicity using the MAP design, and obtained high-titer and -affinity anti-heparinase antibodies in vivo $(15,16)$.

The biological characteristics of TNF- $\alpha$ are not species-specific; indeed, the murine (m)TNF- $\alpha$ is highly homologous to human TNF- $\alpha$ and has a similar tertiary structure (17). Its precursor has 235 amino acid residues containing a signal peptide with 79 amino acids. In the current study, the mTNF- $\alpha$ B cell epitope was predicted using several bioinformatics techniques based on the primary structure of mTNF- $\alpha$, and associated with the interleukin-1 $\beta$ (IL-1 $\beta$ ) 163-171 universal helper T-cell epitope peptide (18); then, the eight-branched MAP design was used to synthesize a complex MAP vaccine. Next, the immunological effects of the produced vaccine were assessed in vitro and in vivo. Additionally, the impact of the vaccine on disease active index (DAI), serum diamine oxidase (DAO) levels, protein levels of colonic tissue claudin 1 and other tight junction proteins, myeloperoxidase (MPO) activity, and epithelial ultrastructural injuries were assessed in BALB/c mice with UC. The aim of the present study was to provide a basis for the development of novel TNF- $\alpha$ vaccines against UC.

\section{Materials and methods}

Materials. The following materials were used: mTNF- $\alpha$ recombinant protein (eBioscience; Thermo Fisher Scientific, Inc., San Diego, CA, USA); polyclonal rabbit anti-mouse TNF- $\alpha$ full length protein antibodies (cat. no. 17590-1-AP; Proteintech Tech Group, Inc., Chicago, IL, USA); Freund's complete and incomplete adjuvants (Sigma-Aldrich; Merck KGaA, Darmstadt, Germany), horseradish peroxidase-conjugated goat anti-rabbit immunoglobulin (IgG) (cat. no. BA1003; Wuhan Boster Biological Technology, Ltd., Wuhan, China); anti-claudin 1 (cat. no. EPRR18871), -occludin (cat. no. EPR8208), -ZO1 (cat. no. EPR19945-296; Abcam, Cambridge, UK) and GAPDH (cat. no. 10494-1-AP; Proteintech Tech Group, Inc.) antibodies, a western blotting chemiluminescence kit (Kangchen Bio-tech Inc., Shanghai, China); lactate dehydrogenase (LDH) assay kit (Jiancheng Bioengineering Institute, Nanjing, China) and protein markers (Bio-Rad Laboratories, Inc., Hercules, CA, USA) were used. Mouse H22 hepatocarcinoma cells, which highly express mTNF- $\alpha$, were obtained from Huazhong University of Science and Technology (Wuhan, China), and mouse L929 fibroblasts from American Type Culture Collection (Manassas, VA, USA). BALB/c mice ( $n=48,3-4$ weeks old, 15-18 g; $n=30$, 4-5 weeks old; $18-20 \mathrm{~g}$ ) were purchased from Shanghai Sipper-BK Laboratory Animal Co. Ltd. [production license no. SCXK
(Shanghai) 2013-0016] and housed in the barrier environment of Experimental Animal Center in Zhejiang Province [use license no. SYXK (Zhejiang) 2014-0008]. The mice were kept in Plexiglas ${ }^{\circledR}$ cages placed on a laminar flow ultra-clean rack. A total of 3-4 mice were housed in each cage at a constant temperature $\left(25-27^{\circ} \mathrm{C}\right)$ and humidity (45-50\%), under a 12-h light/dark cycle with free access to food and sterilized water. Water and food consumption volumes were recorded daily. The animal studies were approved by the Ethics Committee of Zhejiang Chinese Medical University (approval no. ZSLL-2011-95; Hangzhou, China). Furthermore, $0.3 \%$ sodium pentobarbital was administered intraperitoneally at a dose of $30 \mathrm{mg} / \mathrm{kg}$ to minimize perioperative animal suffering. All animals survived till the end of the experiments. At the end of the experiments (after 7 or 10 weeks), all animals were anesthetized intraperitoneally with $0.3 \%$ sodium pentobarbital $(60 \mathrm{mg} / \mathrm{kg})$, and then euthanasia was performed by cervical dislocation.

\section{Complex vaccine preparation}

Epitope prediction. The antigenic epitopes of mouse TNF- $\alpha$ were predicted using Predicting Antigenic Peptides software (http://www.mif.dfci.harvard.edu/Tool/antigenic.pl; Harvard University, Cambridge, MA, USA), using the semi-empirical Kolaskar and Tongaonkar methods (19), to initially determine the B cell epitope region of mTNF- $\alpha$. The 235 amino acid sequences of the mTNF- $\alpha$ sequence, particularly those within the regions determined using the Predicting Antigenic Peptides software, were then analyzed by DNAStar 7.1 (DNASTAR, Inc., Madison, WI, USA) and the BcePred online prediction tool (http://www.imtech.res.in/raghava/bcepred/) to evaluate the physical and chemical properties of epitope region proteins, including hydrophilicity, accessibility, plasticity and antigenicity. Finally, the most likely amino acid sequence of the predominant mTNF- $\alpha$ B-cell epitope was selected.

MAP synthesis. MAP synthesis was performed by Hangzhou Peptide Biochemical Co., Ltd. on an ABI431A peptide synthesizer (Perkin-Elmer, Inc., Waltham, MA, USA) using the eight-branched MAP resin. The eight-branched MAP core structure was synthesized based on the Fmoc synthesis scheme (20). The predicted predominant mTNF- $\alpha$ B-cell epitope peptide and IL-1 $\beta$ universal helper T-cell epitope peptide VQGEESNDK were synthesized and connected by lysine to form a complex peptide. Then, the resulting molecule was coupled with eight amino terminals of the eight lysine residues located at the end of the MAP core structure. In accordance with amino acid sequences of peptides, the amino acid was sequentially connected one by one from the carboxy-terminus to the amino terminal end. The collected sample was then subjected to low pressure rotary evaporation at a constant temperature $\left(4^{\circ} \mathrm{C}\right)$ and freeze-dried overnight, to yield the lyophilized eight-branched TNF- $\alpha$ B cell epitope-IL-1 $\beta$ T helper epitope complex MAP vaccine. Additionally, an eight-branched MAP-VQGEESNDK sequence was also synthesized in the same way, as a control, and simply consisted of eight $\mathrm{T}$ helper peptide.

\section{Immunological effects of the complex MAP vaccine}

Binding affinity. The binding affinity of the complex MAP vaccine to the mTNF- $\alpha$ whole protein antibody was 
determined using $10 \mathrm{mg} / \mathrm{l}$ of the predicted epitope peptide, the complex MAP vaccine, MAP-VQGEESNDK or the mTNF- $\alpha$ recombinant protein to coat 96-well ELISA plates (100 $\mu 1 /$ well) overnight at $4^{\circ} \mathrm{C}$. Following blocking with $1 \%$ bovine serum albumin (BSA; cat. no. LS000290; US Biochemical Corp., Cleveland, OH, USA), $0.06 \mu \mathrm{g} / 100 \mu \mathrm{l} /$ well of the rabbit anti-mouse full-length TNF- $\alpha$ antibody was added, with 1:4,000 normal BALB/c mouse serum (Zhejiang Chinese Medical University, Hangzhou, China) serving as a control. The absorbance of the plates was measured at a wavelength of $490 \mathrm{~nm}$ using the Imark Micoplate Absorbance Reader 13550 (Bio-Rad Laboratories Inc.), the experiment was repeated three times.

Immunogenicity of the complex MAP vaccine. A total of 48 male BALB/c mice (21) (3-4 weeks old, 15-18 g) were randomly divided into four groups of 12: Epitope peptide, complex MAP vaccine, mTNF- $\alpha$ recombinant protein and MAP-VQGEESNDK. The mTNF- $\alpha$ recombinant group served as the control. The four peptides were used as immunogens. The first immunization used Freund's complete adjuvant, while boost vaccinations included Freund's incomplete adjuvant. The initial and boost doses in the epitope peptide, the MAP-VQGEESNDK and the complex MAP vaccine groups were $0.2 \mathrm{mg} / \mathrm{mouse} ; 20 \mu \mathrm{g} /$ mouse was used in the mTNF- $\alpha$ recombinant protein group. Each immunogen was dissolved in $0.5 \mathrm{ml}$ of PBS and emulsified with $0.5 \mathrm{ml}$ Freund's adjuvant prior to use for multiple subcutaneous injections into the backs of the animals. Two weeks after each vaccination, orbital blood samples were collected, and the mice were euthanized. A total of five injections were administered at 2-week intervals and blood was collected six times for serum isolation. Next, antibody titers in serum were determined using standard indirect enzyme linked immunosorbent assay (ELISA). The 96-well ELISA plates were coated with $10 \mathrm{mg} / \mathrm{l}$ epitope peptide, complex MAP vaccine, MAP-VQGEESNDK and mTNF- $\alpha$ recombinant whole protein at $100 \mu \mathrm{l} /$ well overnight at $4^{\circ} \mathrm{C}$. After blocking with $1 \% \mathrm{BSA}$ for $1 \mathrm{~h}$ at $37^{\circ} \mathrm{C}$, the sections were incubated with polyclonal rabbit anti-mouse TNF- $\alpha$ full length protein antibodies (dilution, $1: 4,000$ ) for $1 \mathrm{~h}$ at $37^{\circ} \mathrm{C}$ and then horseradish peroxidase-conjugated goat anti-rabbit IgG (dilution, 1:4,000) for $1 \mathrm{~h}$ at $37^{\circ} \mathrm{C}$ to test the immunogens; the pre-immunized serum was used as a negative control. The ratio of the absorbance of the test sample to that of the negative control was calculated, and a value $>2.1$ indicated a positive signal; the highest dilution that yielded a positive reaction was considered the antibody titer of the sample.

Western blot analysis. Following centrifugation, $1 \times 10^{9}$ murine $\mathrm{H} 22$ cells and $1 \mathrm{ml}$ of ice-cold suspension buffer was added. The cells were then dispersed with a vortex oscillator for $5 \mathrm{~min}$ before centrifugation at $1,006 \mathrm{xg}$ for $5 \mathrm{~min}$ at $4^{\circ} \mathrm{C}$. The supernatant was discarded, and an equal volume of $2 \mathrm{X}$ SDS was immediately added to the extract total protein. SDS-PAGE was performed on a 5\% gel, and a total of $35 \mathrm{mg}$ protein per lane was analyzed, with the commercial mTNF- $\alpha$ recombinant protein used as the positive control and the pre-immunized mouse serum serving as a negative control. Western blotting was performed following the manufacturer's protocol. Immunoreactive bands were detected using chemiluminescence reagent (Shanghai Kangchen Bio-tech Inc.).

TNF- $\alpha$ inhibitory effects of MAP antiserum. Growing L929 cells were adjusted to a density of $5 \times 10^{7}$ cells $/ \mathrm{ml}$ and $100 \mu \mathrm{l}$ of the cells was inoculated in each well of 96-well plates for $12 \mathrm{~h}$. The antiserum and mTNF- $\alpha$ in each group were diluted with $0.5 \mathrm{mg} / \mathrm{l}$ actinomycin $\mathrm{D}$, which increases the sensitivity of L929 cells to TNF- $\alpha$ cytotoxicity. mTNF- $\alpha$ at $400 \mu \mathrm{g} / 1$ was added into each well, which contained $10 \mu l$ of the epitope peptide antiserum, the complex MAP vaccine antiserum, the MAP-VQGEESNDK antiserum and the mTNF- $\alpha$ recombinant protein antiserum at a dilution of 1:500. In the control wells, only the cell culture medium containing $0.5 \mathrm{mg} / \mathrm{l}$ actinomycin D was added. After $24 \mathrm{~h}$, supernatants were collected, the absorbance was measured in triplicate at $450 \mathrm{~nm}$ on a microplate reader (BioTek Instruments, Inc., Winooski, VT, USA) and the cytotoxicity (\%) of L929 cells were quantified by the LDH activity according to the following formula: [(LDH) E-(LDH)TS]/[(LDH)TSmax-(LDH)TS] x100. Where, (LDH) $\mathrm{E}$ is the level of experimental LDH release, (LDH)TS is the level of target spontaneous LDH release and (LDH)TSmax is the target maximum level of LDH release. Spontaneous LDH release was evaluated by incubating target cells free of mTNF- $\alpha$, while maximum release was assessed by the presence of maximum mTNF- $\alpha$.

Therapeutic effects of the complex MAP vaccine in UC mice Immunization and modeling. A total of female BALB/c mice (4-5 weeks old; $18-20 \mathrm{~g}$ ) were divided into three groups $(\mathrm{n}=10)$ according to body weight: The immunization, the UC model and the blank control groups. There was no significant difference in body weight among groups.

In the immunization group, mice were multi-point immunized subcutaneously with the complex MAP vaccine on day 1 . The immunization dose was $0.2 \mathrm{mg} /$ mouse with Freund's complete adjuvant, followed by two booster immunizations with Freund's incomplete adjuvant (22). The adjuvant and antigen were fully mixed at a ratio of 1:1 and administered every 2 weeks. BALB/c mice were treated with equal volumes of PBS in the UC model group, and the immunization scheme was the same as in the immunization group.

The UC model was established by free access to $5 \%$ dextran sulfate sodium (DSS) solution. After 4 weeks (corresponding to the third immunization), mice in the immunization and UC model groups were administered DSS for 7 days, while mice in the blank control group drank distilled water.

DAI scoring. After drinking the DSS solution, alterations to mouse weight and stool properties were observed daily, and fecal occult blood was detected using the test paper method (23). A total of 3 weeks from the start of DSS administration, data were scored as indicated in Table I. Scores of weight loss, stool traits and fecal blood were added to determine the mean DAI score for each mouse; thus, evaluating the disease status of the animals (23). Meanwhile, antibody titers in mice were detected weekly.

Determination of serum DAO content. Mice were intraperitoneally injected with $30 \mathrm{mg} / \mathrm{kg} 1 \%$ sodium pentobarbital to 
Table I. Mouse DAI scoring table.

\begin{tabular}{lcll}
\hline Score & Weight loss $(\%)$ & Stool traits $^{\mathrm{a}}$ & \multicolumn{1}{c}{ Blood stool } \\
\hline 0 & None & Normal & Normal \\
1 & $1-5$ & Loose & Occult blood (+) \\
2 & $5-10$ & & \\
3 & $10-15$ & Diarrhea & Visible blood \\
4 & $>15$ & & \\
\hline
\end{tabular}

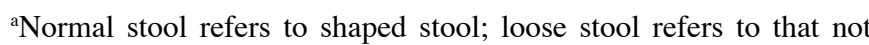
sticking to the anus and semi-shaped; diarrhea refers to dilute watery stool that sticks to the anus. DAI, disease active index.

induce deep anesthesia. The animals were sacrificed 3 weeks after drinking DSS. Specimens were collected after mice had fasted for $12 \mathrm{~h}$ with free access to water. Eyes were removed and orbital blood samples were collected repeatedly with a siphon to obtain $\sim 1 \mathrm{ml}$ of blood, which was added to centrifuge tubes containing $1 \%$ heparin. The blood samples were centrifuged at $1,006 \mathrm{x} \mathrm{g}$ for $5 \mathrm{~min}$ at $4^{\circ} \mathrm{C}$ to obtain serum. Serum DAO content was determined using the double antibody sandwich avidin biotin peroxidase complex (ABC)-ELISA (cat. no. ab155458; Abcam) method (24). Optical density values were measured at $450 \mathrm{~nm}$ and DAO concentrations were determined using a standard curve.

Observation of bacterial translocation in mesenteric lymph nodes. After blood collection, the mice were dissected. Approximately $200 \mathrm{mg}$ of mesenteric lymph nodes were extracted under aseptic conditions and homogenized in a sterile grinder. After centrifugation at $300 \mathrm{x}$ g for $5 \mathrm{~min}$ at $4^{\circ} \mathrm{C}$, $100 \mu \mathrm{l}$ of the supernatant was inoculated on a common blood agar dish for quantitative culturing. The culture plates were incubated at $37^{\circ} \mathrm{C}$ for $48 \mathrm{~h}$ and the mean number of colonies in the three groups were compared.

Expression levels of tight junction proteins. Immunoblotting was used to assess the expression levels of tight junction proteins, including claudin 1, occludin and ZO1, in colon tissue specimens. Approximately $0.5 \mathrm{~cm}$ of mouse colon was added to the protein extraction buffer, homogenized with a homogenizer for $30 \mathrm{~min}$ at $0^{\circ} \mathrm{C}$ and centrifuged at $21,910 \mathrm{x} \mathrm{g}$ for $15 \mathrm{~min}$ at $4^{\circ} \mathrm{C}$. The supernatants were collected, and protein amounts were quantified using the Lowry method.

Equal amounts of total protein $(30 \mu \mathrm{g} / \mathrm{lane})$ were separated by SDS-PAGE on a $5 \%$ gel; the protein bands were transferred onto nitrocellulose membranes. Non-specific binding was blocked with $5 \%$ milk, which was incubated with the membranes for $2 \mathrm{~h}$ at $4^{\circ} \mathrm{C}$. The membranes were then incubated with anti-claudin 1, anti-occludin, anti-ZO1 antibodies (dilution, 1:1,000) and anti-GAPDH (dilution, 1:10,000) at $4^{\circ} \mathrm{C}$ overnight. This was followed by incubation with a horseradish peroxidase-conjugated secondary antibody (dilution, 1:10,000) for $2 \mathrm{~h}$ at room temperature. Detection was performed with an enhanced chemiluminescence kit. GAPDH was used as an internal control. BandScan 5.0 software (Glyko Inc., Novato, CA, USA) was used to analyze the gray scale values of different bands.
Histological damage pathology scoring. Approximately $1 \mathrm{~cm}$ of distal colon was fixed in $10 \%$ formaldehyde. This was followed by conventional paraffin embedding, slicing (4- $\mu$ m-thick sections), and hematoxylin and eosin (H\&E) staining. Histological changes, including colonic mucosal ulcers, interstitial edema, and nucleated cell infiltration were observed using a Nikon Eclipse 400 microscope (Nikon Corporation, Tokyo, Japan) at a magnification of $\mathrm{x} 200$. The severity of colonic tissue inflammation, depth of lesions, and crypt and epithelial damage were evaluated by two senior pathologists. The degree of histological damage was expressed by the histological index (HI; Table II) (25).

Ultrastructure of mucous epithelium. Approximately $0.5 \mathrm{~cm}$ of distal ileum was fixed in $2.5 \%$ glutaraldehyde at $4^{\circ} \mathrm{C}$ for $6 \mathrm{~h}$. Electron microscope specimens were routinely prepared, and stained with uranium acetate and lead citrate. All the slices were sectioned using the LKB-V type ultramicrotome (LKB Bromma; GE Healthcare Life Sciences, Little Chalfont, UK). The ultrastructural of mucous epithelium was observed under the JEM-1010 type transmission electron microscope (TEM; JEOL, Ltd., Tokyo, Japan).

Determination of MPO activity in the colon tissue. MPO is an enzyme in neutrophil azurophilic granules, and its activity reflects the number and activity of neutrophils. To determine MPO activity, mouse colon tissue was accurately weighed. Then, homogenization medium was added to the tissue based on a tissue weight $(\mathrm{g})$ :volume $(\mathrm{ml})$ ratio of $1: 19$; the resulting solution was mixed to prepare a $5 \%$ tissue homogenate mixture. According to the manufacturer's protocol of MPO assay kit (cat. no. A044; Jiancheng Bioengineering Institute), the reagents were added in order. Briefly, the prepared tissue mixture was added to $50 \mathrm{mmol} / \mathrm{l}$ phosphate buffer $(\mathrm{pH} 6.0)$ containing $0.5 \%$ hexadecyltmethyl-ammonium bromide. The sample was then centrifuged at $16,099 \mathrm{x} \mathrm{g}$ for $20 \mathrm{~min}$ at $4^{\circ} \mathrm{C}$ and the supernatant was used for the MPO assay by utilizing $0.0005 \%$ hydrogen peroxide as a substrate. Distilled water was used to as the blank control and absorbance was then determined at $460 \mathrm{~nm}$. Results were expressed as units per gram weight $(\mathrm{U} / \mathrm{g})$ of wet tissue.

Statistical analysis. Statistical analysis was performed using the SPSS 17.0 software (SPSS, Inc.). Quantitative data are presented as mean \pm standard deviation. Multiple samples were compared by one-way ANOVA with the Student-Newman-Keuls post-hoc test. $\mathrm{P}<0.05$ was considered to indicate a statistically significant difference.

\section{Results}

Prediction of $m T N F-\alpha$ epitopes and complex MAP vaccine synthesis. The amino acid sequence of the $\mathrm{mTNF}-\alpha$ protein was obtained from Genbank (Fig. 1). From the results obtained with the Predicting Antigenic Peptides software, the mouse TNF- $\alpha$ B-cell epitopes were located within amino acids 10-20, 46-103, 111-125 and 146-153. The selected region of the mTNF- $\alpha$ sequence was analyzed by DNAStar and Bcepred software. The results showed that the LTLRSSSQNSSDKPV peptide in the gray background composed of amino acids 78-92 had the 
Table II. Standard of histological index.

\begin{tabular}{lccc}
\hline Score & Inflammation & Depth of the lesion & Crypt destruction \\
\hline 0 & None & None & None \\
1 & Mild & Mucosal muscularis & $1 / 3$ basement Crypt destruction \\
2 & Severe & Muscular layer & $2 / 3$ basement Crypt destruction \\
3 & - & Serous layer & Only complete surface epithelium \\
4 & - & - & All epithelial damage \\
\hline
\end{tabular}

\begin{abstract}
MSTESMIRDVELAEEALPQKMGGFONSRRCLCLSLFSFLLVAGATTL FCLLNFGVIGPQRDEKFPNGLPLISSMAQTLTLRSSSONSSDKPVAHV VANHQVEEQLEWLSQRANALLANGMDLKDNQLVVPADGLYLVYS QVIFKGQGCPDY $L T H T V S R F A I S Y Q E K V N L S A V K S P C P K D T P E G$ AELKPWYEPIYLGGVFQLEKGDQLSAEVNLPKYLDFAESGQVYFGVI AL
\end{abstract}

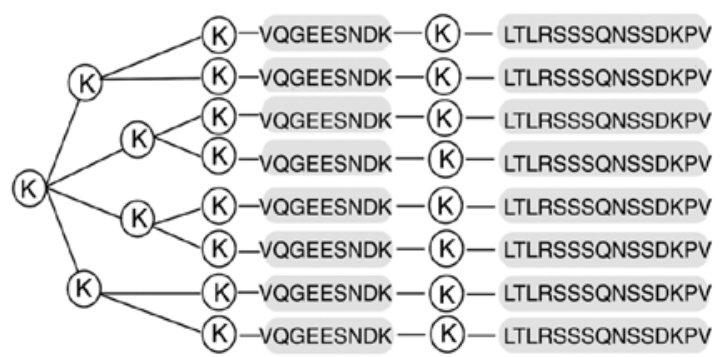

Figure 1. Amino acid sequence of $\operatorname{mTNF}-\alpha$ and diagram of the 8-branched complex MAP vaccine structure. Gray-labeled amino acid was the dominant epitope peptide of mTNF- $\alpha$. The predicted mTNF- $\alpha$ predominant B-cell epitope peptide LTLRSSSQNSSDKPV and IL-1 $\beta$ universal helper T-cell epitope peptide VQGEESNDK were synthesized and connected to form a complex peptide. Then, the resulting molecule was coupled with eight amino terminals of the eight lysine residues located at the end of the MAP core structure. mTNF- $\alpha$, murine tumor necrosis factor- $\alpha$; IL- $1 \beta$, interleukin- $1 \beta$; MAP, multiple antigenic polypeptide.

best hydrophilicity, accessibility and plasticity. Moreover, it was located within the protein extension structure or irregular curl structure, constituting the most likely dominant B-cell epitope. Therefore, the TNF- $\alpha$ B epitope-IL- $1 \beta$ helper T-cell epitope complex MAP vaccine based on the latter predicted amino acid sequence was synthesized. Fig. 1 depicts the structure of the complex MAP vaccine, which was purified by high pressure liquid chromatography, to achieve a purity of $\geq 95 \%$.

Binding affinity of the complex MAP vaccine to a TNF- $\alpha$ whole protein antibody. The binding affinity was detected by indirect ELISA, and the results demonstrated that the synthesized complex MAP vaccine had strong binding affinity with the mTNF- $\alpha$ whole protein antibody in vitro. Its binding affinity with the antibody was weaker compared with that of the mTNF- $\alpha$ recombinant protein, but stronger compared with that of the epitope oligopeptide or MAP-VQGEESNDK $(\mathrm{P}<0.01$; Fig. 2A). These findings indicated that the eight-branch MAP design and the insertion of the IL- $1 \beta$ helper T-cell epitope peptide significantly enhanced the antigenicity of the predicted mTNF- $\alpha$ B-cell epitope peptide LTLRSSSQNSSDKPV, and the mTNF- $\alpha$ B cell epitope oligopeptide or helper T-cell epitope polypeptide, VQGEESNDK, did not have binding affinity with the mTNF- $\alpha$ whole protein antibody when compared with negative serum control.

Dynamic changes of antibody titers. Specific antibodies were detected from 2 weeks after the first immunization with the complex MAP vaccine. Antibody titers increased rapidly to $\sim 1: 119,000$ after 4 weeks. After 6 weeks, antibody titers plateaued with a value of $1: 168,000$. In week 8 , antibody titers reached its peak value, which declined steadily to $1: 173,000$ by week 10 . The epitope peptide, $\mathrm{mTNF}-\alpha$ recombinant protein and MAP-VQGEESNDK groups produced no detectable specific antibodies (Fig. 2B), and the IL-1 $\beta$ helper T-cell epitope peptide and MAP vaccine groups showed no cross reaction. The aforementioned finding showed that a high titer of antibody can be induced by the complex MAP vaccine.

Specificity of the antibody verified by western blot analysis. Total protein from $\mathrm{H} 22$ cells was incubated with the immunized epitope peptide, complex MAP vaccine, MAP-VQGEESNDK and commercial mTNF- $\alpha$ recombinant protein antibody antiserum samples. A rabbit anti-mouse TNF- $\alpha$ antibody was used as a positive control; GAPDH was used as internal reference gene. Incubation with the commercial mTNF- $\alpha$ recombinant protein antibody produced a thick band of $\sim 17 \mathrm{kDa}$ and the band in the MAP vaccine group was also evident. According to the specification of the commercial antibody, $17 \mathrm{kDa}$ corresponds to the mTNF- $\alpha$ protein. The mTNF- $\alpha$ recombinant protein, MAP-VQGEESNDK and epitope peptide groups did not show overt bands in the corresponding location (Fig. 2C). The aforementioned results suggested that the immunized antibody in the complex MAP vaccine specifically binds to the TNF- $\alpha$ protein.

Effects of various antisera on TNF- $\alpha$ activity. The viability of L929 cells with recombinant mTNF- $\alpha$ was detected using the LDH release assay to assess the inhibitory effects of the antisera on the biological activity of mTNF- $\alpha$. The results showed that the cytotoxicity in the epitope peptide, complex MAP vaccine, mTNF- $\alpha$, MAP-VQGEESNDK antiserum groups and the control group were 44.59, 23.47, 53.16, 47.93 and 53.80 , respectively. This indicated that cytotoxicity measured by the LDH activity was significantly lower in the complex MAP vaccine antiserum group compared with in the other four groups $(\mathrm{P}<0.01)$ and that TNF- $\alpha$ activity in the complex MAP vaccine group was primarily inhibited by the specific antibody contained in the antiserum. Compared with the control group, the MAP-VQGEESNDK, epitope peptide or mTNF- $\alpha$ antiserum groups showed no significant differences (Fig. 2D). 


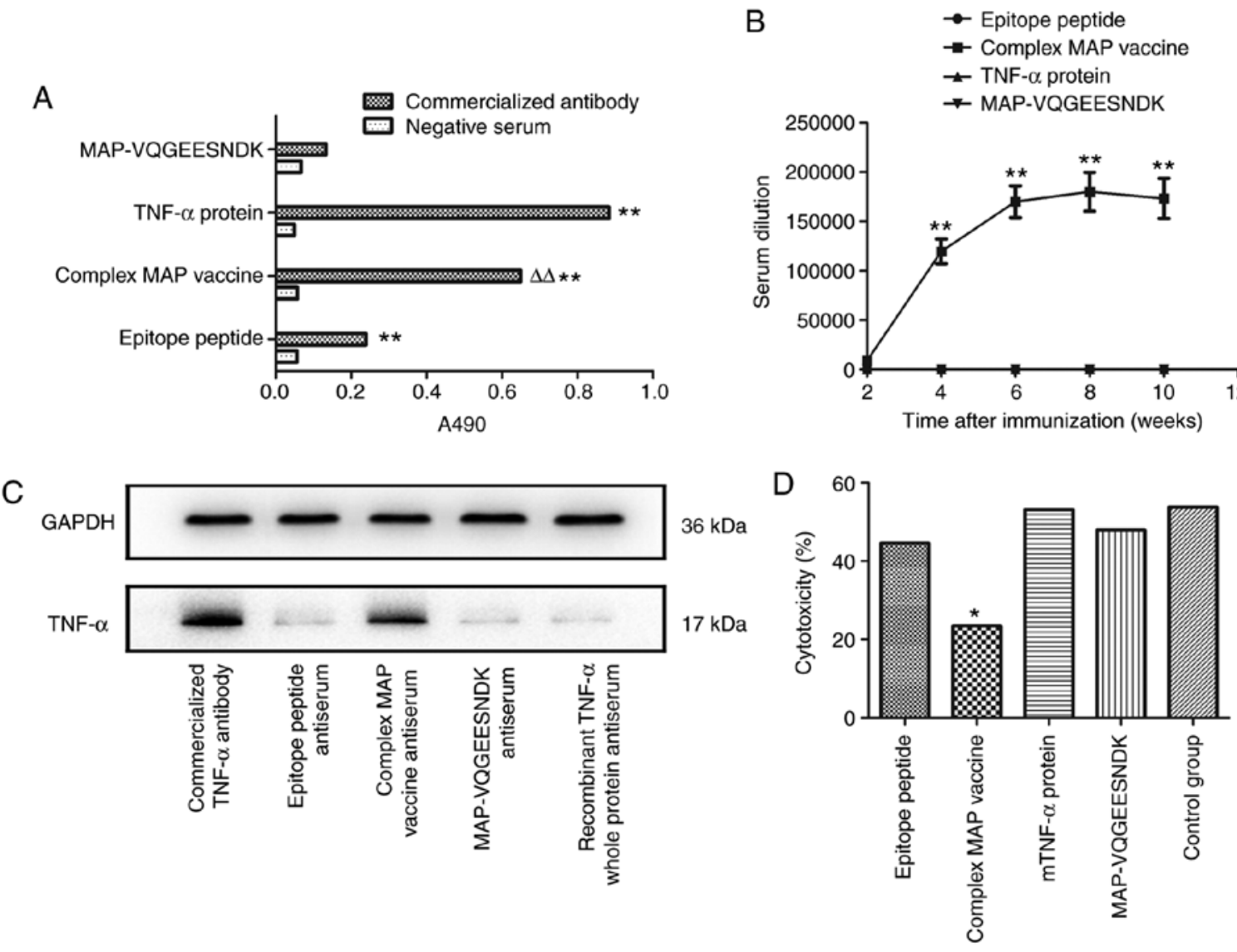

Figure 2. Immunological effects of the complex MAP vaccine. (A) Binding affinity with the commercialized anti-mTNF- $\alpha$ antibodies. The binding affinity of the complex MAP vaccine with mTNF- $\alpha$ whole protein antibodies was weaker compared with that of murine TNF- $\alpha$ protein, but significantly stronger compared with that of the MAP-VQGEESNDK or epitope oligopeptide. Mean \pm SD. $n=3$. ${ }^{* *} \mathrm{P}<0.01$ vs. negative serum; ${ }^{\Delta \Delta} \mathrm{P}<0.01$ vs. TNF- $\alpha$ protein, MAP-VQGEESNDK or epitope peptide. (B) Dynamic changes of anti-MAP antibody titers in murine serum. Mean $\pm \mathrm{SD} . \mathrm{n}=3$. ${ }^{* *} \mathrm{P}<0.01$ vs. other three groups. (C) Specificity of murine antisera against MAP as detected by Western blotting. (D) Effects of antisera on bioactivity of mTNF- $\alpha$. Recombinant mTNF- $\alpha$ and antisera of various groups were co-cultured with L929 cells, followed by LDH release assay to assess the cytotoxicity of mTNF- $\alpha$ to L929. The results showed that the cytotoxicity in the eight-branched complex MAP vaccine antiserum group was significantly lower compared with the value of any other group $(\mathrm{P}<0.01)$, and there was no difference among other four groups $(\mathrm{P}>0.05)$. The TNF- $\alpha$ activity in the vaccine group was primarily inhibited by the specific antibody. Mean \pm SD. $n=3$. ${ }^{*} \mathrm{P}<0.01$ vs. other four groups. mTNF- $\alpha$, murine tumor necrosis factor- $\alpha$; MAP, multiple antigenic polypeptide; LDH, lactate dehydrogenase.

Effects of vaccination on DAI scores of UC mouse. The DAI score of the blank control group was 0 throughout the experiment. The DAI score of the UC model group was 0 for the first 2 days of 5\% DSS consumption. DAI scores gradually increased with the duration of DSS consumption, peaking at 9.12 on day 7. After DSS termination, the values began to decline and dropped to 5.69 on day 21. The DAI score of the immunization group was 0 for the first 3 days of 5\% DSS consumption. It gradually increased with the duration of DSS consumption and peaked at 6.92 on day 7 ; the values decreased to 1.03 on day 21 (Fig. 3). Antibody titers gradually increased over 3 weeks. As the antibody titers increased, the difference between the DAI scores of the immunized and UC groups gradually increased. The results showed that DSS induced less colonic damage in the immunization group, and repair of the colonic damage in the immunization group was faster and more efficient compared with the UC model group.

Serum DAO levels in immunized mice. To assess the permeability of the intestinal mucosa in mice, serum DAO levels in mice were measured using the double antibody sandwich ABC-ELISA method at 7 weeks after the first immunization.
Serum DAO levels in UC model group were $3.38 \pm 0.54$ and $1.56 \pm 0.37 \mathrm{U} / \mathrm{ml}$ in the immunization group, a statistically significant difference was identified between the two groups $(\mathrm{P}<0.05)$. Compared with the blank control group $(1.06 \pm 0.29 \mathrm{U} / \mathrm{ml})$, no significant difference was identified in the immunization group (Fig. 4A). These results indicate that the complex MAP vaccine can protect intestinal mucosal cells in mice.

Bacterial growth in mesenteric lymph nodes after immunization. Following blood collection in week 7 , the mice were dissected. Mesenteric lymph nodes were collected and homogenized; the colonies were then counted following inoculation and culture. The permeability of the intestinal mucosa was evaluated by comparing the mean number of colonies among the three groups of mice. The results showed that bacterial translocation did not occur in the mesenteric lymph nodes in the blank control group. The colony count of mesenteric lymph nodes in the UC model group was $3.1 \pm 0.88$; a significantly reduced bacterial count was obtained in the immunization group $(1.2 \pm 0.67$; $\mathrm{P}<0.05$; Fig. 4B). The results showed that the complex MAP vaccine can reduce intestinal bacterial translocation to a certain extent. 


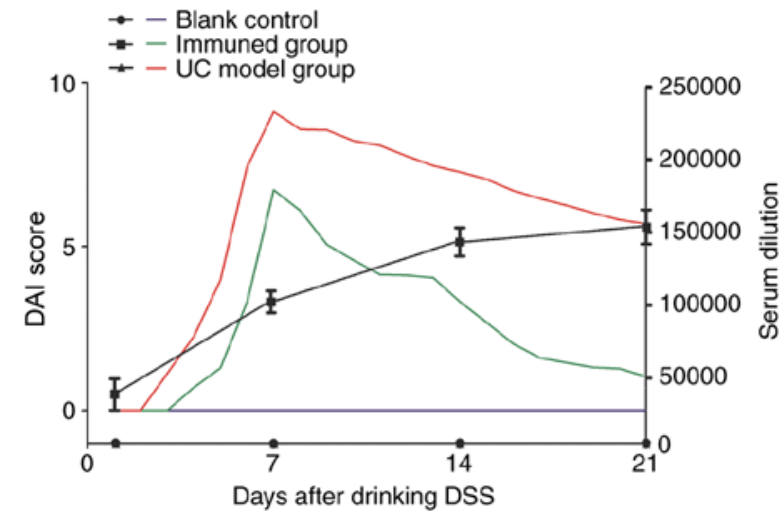

Figure 3. Mice DAI scores and serum antibody titers after drinking DSS. From the beginning of DSS administration, DAI scores were recorded daily and serum antibody titers weekly for a total of 3 weeks. Throughout the whole process, the DAI score of the blank control group was 0; No antibody titers were detected in the blank control group and the UC model group. The DAI score of the UC model control group was 0 the first 2 days of 5\% DSS consumption, reaching the highest value of 9.12 on day 7 ; the values gradually decreased after DSS discontinuation. On day 21, scores averaged 5.69. The DAI score of the immunization group the first 3 days was 0 , reaching the highest value of 6.92 on day 7 , before declining significantly to 1.03 on day 21 . Antibody titers in the immunization group was $1: 4,900$ on day 1 . Then, the antibody titers increased to $1: 115,000$ on day $7,1: 14,900$ on day 14 and 1:15,100 on day 21. As the antibody titers increases, the difference between the DAI score of the immunization group and the DAI score of the UC model group gradually increases. UC, ulcerative colitis; DSS, dextran sulfate sodium; DAI, disease active index.

Protein expression levels of claudin 1, occludin and ZOI in colon tissue samples after immunization. To evaluate the integrity of the mouse intestinal mucosa, western blot analysis was used to detect the expression levels of claudin1, occludin and ZO1 in colon tissue samples from mice in each group. With GAPDH as an internal reference gene, the ZO1 band was primarily concentrated at $\sim 194 \mathrm{kDa}$, while occludin and claudin 1 were found at $\sim 59 \mathrm{kDa}$, and $20-23 \mathrm{kDa}$, respectively (Fig. 4C). The results, analyzed by the BandScan 5.0 software, showed that the expression levels of claudin 1, occludin and $\mathrm{ZO} 1$ in samples from the UC model group were significantly lower compared with those of the blank control group $(\mathrm{P}<0.05)$. Compared with the blank control group, the expression levels of claudin1, occludin and ZO1 in the immunization group were also decreased, but significantly higher compared those in the UC model group ( $\mathrm{P}<0.05$; Fig. 4D).

Histological damages and HI scores after immunization. In the UC model group (Fig. 5A), H\&E staining of murine colon samples showed incomplete colon mucosa, multifocal ulcers with exudation, primarily damaged epithelial cells and glands, a large number of infiltrated inflammatory cells, evidently decreased goblet cells, submucosal edema, lymph node aggregation, and crypt abscesses. In the blank control group (Fig. 5B), the colonic mucosa was intact with the glands neatly arranged. There was no evident inflammatory cell infiltration. The colonic mucosa of the immunization group was relatively intact, and few shallow ulcers were observed (Fig. 5C). The majority epithelial cells and glands were intact with limited inflammatory cell infiltration; no evident edema was observed in the submucosal layer. The mean HI score of the UC model group was $6.75 \pm 0.62$, which was significantly higher compared with that of the blank control group. The mean HI score of the immunization group was $2.95 \pm 0.37$, which was significantly lower compared with that of the UC model group (Fig. 5D).

Epithelial ultrastructure observed by TEM. In the UC model group (Fig. 5E), evident mucous epithelium injuries were detected by TEM, such as severe cellular edema, atrophic and sparse microvilli, shortened and broadened tight junction complex, and enlarged intercellular gaps. In the blank control group (Fig. 5F), the columnar mucosal epithelia were intact with microvilli neatly and densely arranged, and the tight junction complexes were closely connected without visible gaps. Compared with the UC model group, the ultrastructure of ileac mucous epithelia in the immunization group was relatively normal with slight edema in a few epithelial cells, and the slightly atrophic microvilli were arranged a bit disorderly (Fig. 5G). Intercellular tight junction complexes were evident and intact.

MPO activity in colon tissue homogenates after immunization. To evaluate the degree of colon inflammation in mice, the authors determined MPO activity in colon tissue homogenates from various groups. The results showed that MPO activity in the UC model group was $2.59 \pm 0.23$, which was significantly higher compared with that in the blank control group $(0.89 \pm 0.12)$ and immunization group $(\mathrm{P}<0.01)$, indicating that mice in the UC model group had more severe colon inflammation. MPO activity in colonic homogenates from the immunization group was $1.35 \pm 0.17$; although it was significantly higher compared with that of the blank control group $(\mathrm{P}<0.05)$, this value was significantly lower compared with that of the UC model group $(\mathrm{P}<0.01)$ (Fig. 5H). These findings indicated that the level of colon inflammation in the immunization group was lower compared with that in the UC model group.

\section{Discussion}

TNF- $\alpha$ has attracted increasing attention as a promising autologous molecule for UC treatment $(7,8,26-29)$. The current study aimed to induce high antibody titers in vivo by optimizing the immunization strategies. Using the eight-branched MAP design and inserting the IL-1 $\beta$ helper T-cell epitope peptide significantly enhanced the antigenicity of the predicted TNF- $\alpha$ B-cell epitope peptide LTLRSSSQNSSDKPV. After BALB/c mouse immunization with this complex MAP vaccine, polyclonal antisera were obtained. ELISA showed that the eight-branched MAP could induce high TNF- $\alpha$ antibody titers in vivo, which significantly increased to 119,000 after two immunizations (4 weeks). This time point was selected for subsequent UC experiments to assess the complex MAP vaccine in the alleviation of UC as mice already had more anti-TNF- $\alpha$ antibodies. However, the individual B cell epitope peptide, MAP-VQGEESNDK and TNF- $\alpha$ protein could not induce detectable autoantibodies in vivo, indicating that the designed complex MAP vaccine conformation can effectively break through immune tolerance and greatly enhance the immunogenicity of autoantigens.

As aforementioned, the complex MAP vaccine antiserum showed a clear band at $\sim 17 \mathrm{kDa}$, while the mTNF- $\alpha$ recombinant protein, epitope peptide and MAP-VQGEESNDK antisera 

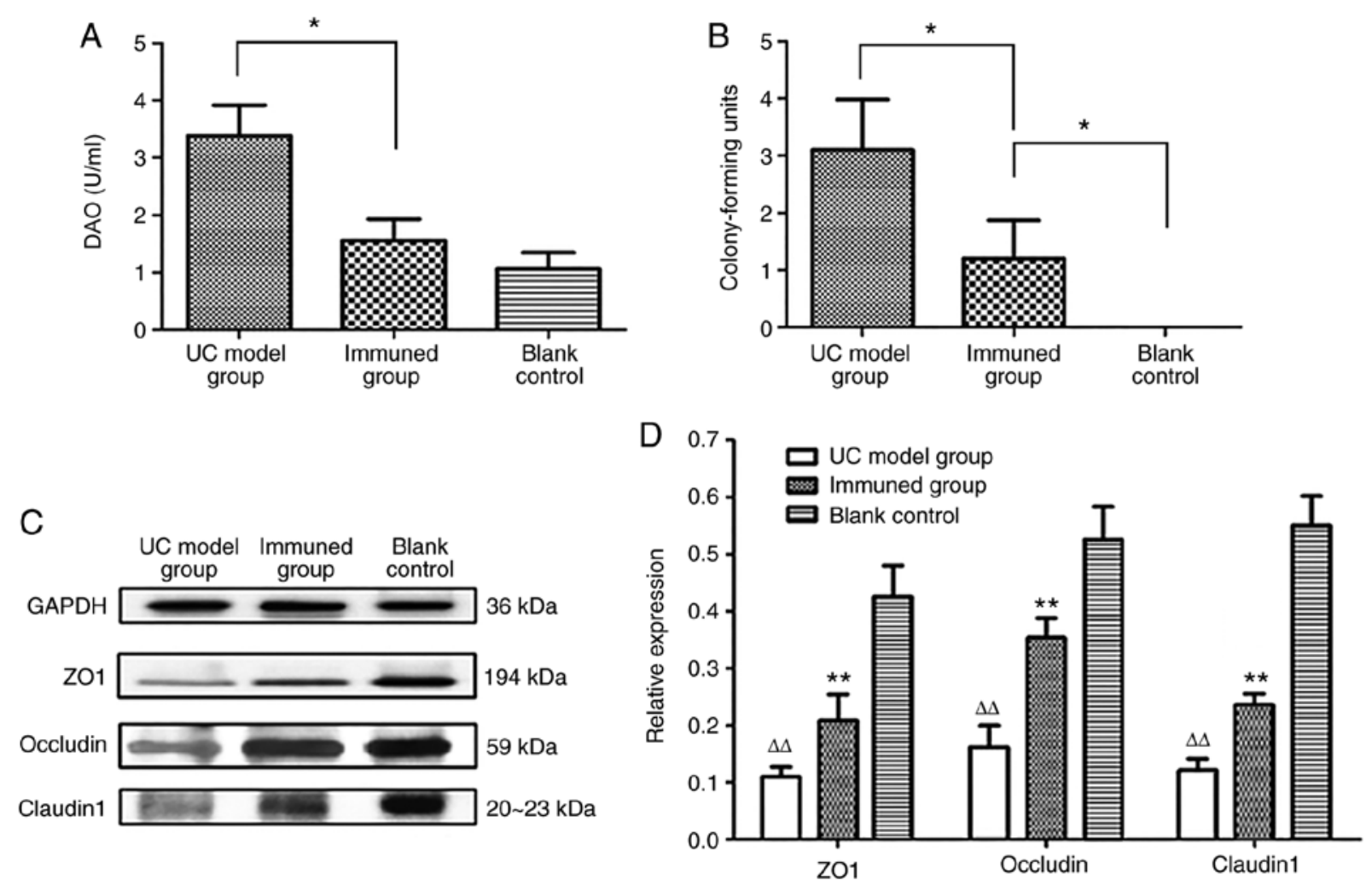

Figure 4. Assessment of intestinal mucosal permeability. (A) The serum DAO content of each group was determined by double antibody sandwich ABC-ELISA. The serum DAO content was $3.38 \pm 0.54 \mathrm{U} / \mathrm{Ml}$ in the $\mathrm{UC}$ model group, $1.56 \pm 0.37 \mathrm{U} / \mathrm{Ml}$ in the immunization group, and $1.06 \pm 0.29 \mathrm{U} / \mathrm{Ml}$ in the blank control group, indicating significantly lower levels in the immunization group compared with the UC model group, ${ }^{*} \mathrm{P}<0.05$, vs. UC model group. The serum DAO level in the immunization group is slightly higher than the blank control group. ${ }^{*} \mathrm{P}<0.05$, vs. blank control group. (B) Mouse colorectal lymph nodes were harvested, and bacterial colonies were counted after culture. The blank control group had no bacterial growth. The colony count of mesenteric lymph nodes in the model group was $3.1 \pm 0.88$, while $1.2 \pm 0.67$ was obtained for the immunization group. The accounts in the latter group was higher than the blank control group, but significantly lower compared with the UC model group. " $\mathrm{P}<0.05$ vs. UC model group or blank control group. (C) Western blotting was used to evaluate the expression levels of claudin1, occludin and ZO1 in colon tissues. With GAPDH as an internal reference, the ZO1 band was primarily concentrated at about $194 \mathrm{kDa}$, while occludin and claudin 1 were found $\sim 59 \mathrm{kDa}$, and 20-23 kDa, respectively. (D) The Western blotting results were also analyzed by the BandScan 5.0 software. Compared with the blank control group, the expression levels of ZO-1, occludin and claudin1 were decreased in the UC model group $\left({ }^{\Delta} \mathrm{P}<0.05\right)$. In the immunization group, the protein expression was lower compared with the blank control group, but was higher compared with the UC model group, which indicating that immunization with the complex MAP vaccine upregulated ZO-1, occludin and claudin1 $\left({ }^{* *} \mathrm{P}<0.05 \mathrm{vs}\right.$. UC model group or blank control). DAO, serum diamine oxidase; UC, ulcerative colitis; MAP, multiple antigenic polypeptide.

showed no evident bands at the corresponding position. These results showed that mTNF- $\alpha$ could not induce a specific humoral immune response in vivo, and the eight-branched MAP design could enhance the immunogenicity of the B-cell epitope LTLRSSSQNSSDKPV, inducing polyclonal antibody specificity binding to TNF- $\alpha$. The authors further assessed the biological effect of the specific antibodies using the LDH release assay, and confirmed that the complex MAP vaccine induced specific antibodies and can significantly inhibit the bioactivity of TNF- $\alpha$.

Chey (30) applied the TNF- $\alpha$ antibody infliximab to 16 patients with UC with $88 \%$ of them demonstrating improved clinical symptoms, colonoscopy and histology results for up to 4 months, indicating that infliximab can alleviate the clinical symptoms of UC. In the UC mouse model, the anti-TNF- $\alpha$ complex MAP vaccine significantly improved the body weight, reduced the symptoms of bloody stool and diarrhea, and decreased DAI scores, in agreement with the therapeutic effects of TNF- $\alpha$ antibodies in passive immunotherapy of patients with UC. Moreover, the immunization group showed a delayed increase in DAI values and lower peak DAI values, and MPO activity in colonic tissue in the immunization group was significantly lower compared with the UC model group. Meanwhile, inflammatory cell infiltration in the mouse colon tissue was reduced, the morphological structures of mucosal epithelial cells and crypts improved, and HI scores decreased. Taken together, these findings demonstrate that the complex MAP vaccine had a certain preventive effect on UC incidence and the occurrence of severe symptoms.

The increased intestinal mucosal permeability of patients with UC is the primary indicator of intestinal mucosal barrier function (31-34). DAO is a highly active intracellular enzyme in the villous cell cytoplasm of the upper layer of the mammalian intestinal mucosa. After damage and necrosis of intestinal mucosal cells, the cells enter into the intestine, leading to increased blood DAO activity (35). Under normal circumstances, the intestinal barrier can effectively block intestinal parasites and endotoxin to the intraluminal displacement (36). When a variety of factors cause intestinal barrier damage, increased permeability, and bacterial translocation may occur. Thus, serum DAO activity and bacterial translocation can reflect the function of the intestinal barrier. Studies have shown that tight junction complexes between intestinal epithelial cells have an important role in maintaining intestinal 

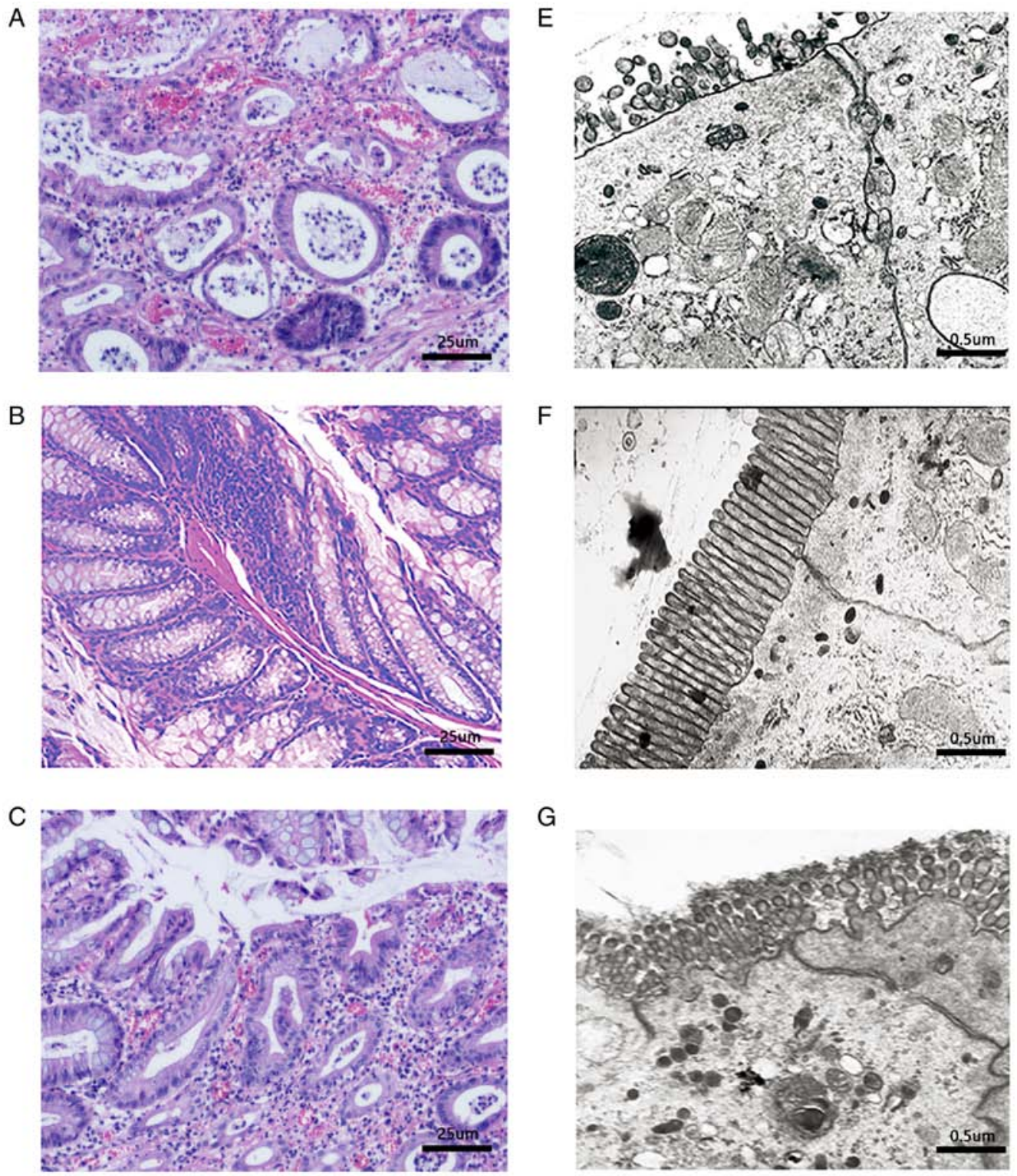

G
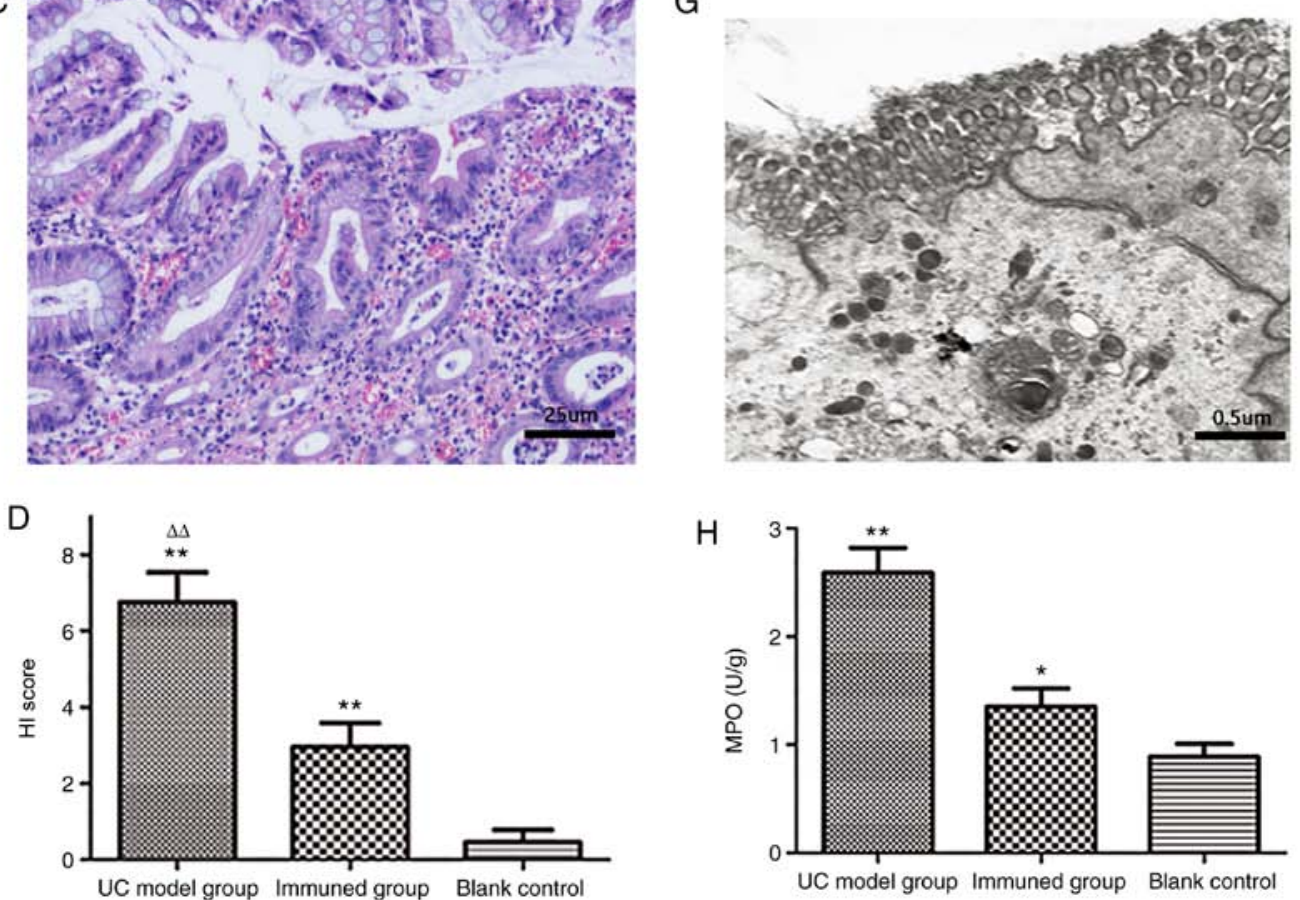

Figure 5. Evaluation of histological injury in murine intestine. (A) The UC model group had no complete colonic mucosa, with massive purulent exudate and mucosal hemorrhage; most goblet cells and glands were damaged, as well as evident inflammatory cells infiltration and crypt abscess (magnification, x200). (B) The colonic mucosa of the blank control group was intact with glands neatly arranged. There was no evident inflammatory cell infiltration (magnification, x200). (C) The colonic mucosa of the immunization group was relatively intact with few inflammatory cells infiltrated; goblet cells were slightly reduced (magnification, x200). (D) Histological damage HI scores. HI scores in the UC model group were $6.75 \pm 0.62$, significantly higher compared with those of the blank control group. HI scores of the immunization group were $2.95 \pm 0.37$, significantly higher compared with those of the blank control group and markedly lower compared with those of the UC model group. ${ }^{* *} \mathrm{P}<0.01$ as vs. blank control, ${ }^{\Delta \Delta} \mathrm{P}<0.01$ as vs. immunization group. (E) Detected under TEM, evident cellular edema, atrophic and sparse microvilli, shortened and broadened tight junction complex and enlarged intercellular gaps were observed in the UC model group (magnification, x10,000). (F) In the blank control group, the columnar mucosal epithelia were intact with microvilli neatly and densely arranged, and the tight junction complexes were closely connected without visible gaps (magnification, $\mathrm{x} 10,000$ ). (G) The ultrastructure of ileac mucous epithelia in the immunization group were relatively normal with slight edema, and the slightly atrophic microvilli were a bit confusedly arranged. Intercellular tight junction complex was rather clear and intact (magnification, x10,000). (H) MPO activity in colon tissue homogenates after immunization. MPO activity in colonic homogenates from the UC model group was significantly higher than that of the blank control and immunization groups. MPO activity in the immunization group was higher than that of the blank control group, but markedly lower compared with that of UC model group. ${ }^{~} \mathrm{P}<0.05$ vs. blank control, ${ }^{* *} \mathrm{P}<0.01$ vs. immunization group or blank control. UC, ulcerative colitis; MAP, multiple antigenic polypeptide; TEM, transmission electron microscope; HI, histological index; MPO, myeloperoxidase. 
mucosal permeability (37-40). The tight junction complexes primarily consist of transmembrane proteins and cytoplasmic proteins, and contain occludin, claudin 1, and ZO1, which reflects the permeability of the intestinal mucosa (38-40). As demonstrated in the present study, serum DAO levels in mice immunized with the complex MAP vaccine were significantly lower compared with those of the UC model group after 3 weeks of DSS consumption. Although the bacterial colony count in lymph nodes of the immunization group was higher compared with that of the blank control group, it was significantly lower compared with that of the UC model group. The expression levels of occludin, claudin1 and $\mathrm{ZO} 1$ in colonic tissue homogenates of UC mice were significantly enhanced by the complex MAP vaccine. The aforementioned findings suggested that the newly-synthesized complex MAP vaccine can improve the permeability of the intestinal mucosa in mice, promoting repair of the intestinal mucosal barrier function.

In the current study, the eight-branched MAP vaccine exhibited good antigenicity and immunogenicity, showing significant anti-UC activity in vivo. The underlying mechanism may be associated with the inhibition of TNF- $\alpha$ biological activity and upregulation of tight junction proteins, as high titers were produced, and polyclonal antibody specificity was determined. However, further in-depth studies are required to determine the optimal vaccine dose, optimize the immune strategy research, optimize the choice of adjuvant, analyze the efficacy of the complex MAP vaccine compared with passive immunization of monoclonal antibodies, and to determine whether the vaccine would induce autoimmune diseases or hypersensitivity reactions. In conclusion, the current study provides theoretical evidence for the further study of the synthesized complex TNF- $\alpha$ B cell epitope MAP vaccine in the treatment of UC.

\section{Acknowledgements}

Not applicable.

\section{Funding}

The present study was supported by the grants of National Natural Science Foundation of China (grant no. 81400682) and Basic Public Welfare Research Project of Zhejiang Province (grant no. LGF18H030009).

\section{Availability of data and materials}

The corresponding author will make available the data generated and analyzed during this study upon reasonable request. All materials used are included in Materials and methods.

\section{Authors' contributions}

YS performed the experiments and contributed to manuscript preparation. WSP and YC performed experiments and analyzed the data. JZ designed and performed experiments; acquired, analyzed, and interpreted the data; and prepared the manuscript. HJW, GQR and LGC analyzed the data and contributed to manuscript preparation. All authors read and approved the final manuscript.

\section{Ethics approval and consent to participate}

The animal studies were approved by the Ethics Committee of Zhejiang Chinese Medical University (approval no. ZSLL-2011-95; Hangzhou, China).

\section{Patient consent for publication}

Not applicable.

\section{Competing interests}

The authors declare that they have no competing interests.

\section{References}

1. Park JH and Brentjens RJ: Adoptive immunotherapy for B-cell malignancies with autologous chimeric antigen receptor modified tumor targeted T cells. Discov Med 9: 277-288, 2010.

2. Castro FV, Al-Muftah M, Mulryan K, Jiang HR, Drijfhout JW, Ali S, Rutkowski AJ, Kalaitsidou M, Gilham DE and Stern PL: Regulation of autologous immunity to the mouse 5T4 oncofoetal antigen: Implications for immunotherapy. Cancer Immunol Immunother 61: 1005-1018, 2012.

3. Steeland S, Libert C and Vandenbroucke RE: A new venue of TNF targeting. Int J Mol Sci 19: E1442, 2018.

4. Kopylov U, Ben-Horin S, Zmora O, Eliakim R and Katz LH: Anti-tumor necrosis factor and postoperative complications in Crohn's disease: Systematic review and meta-analysis. Inflamm Bowel Dis 18: 2404-2413, 2012.

5. Kawalec P, Mikrut A, Wisniewska N and Pilc A: Tumor necrosis factor-alpha antibodies (infliximab, adalimumab and certolizumab) in Crohn's disease: Systematic review and meta-analysis. Arch Med Sci 9: 765-779, 2013.

6. Marchioni RM and Lichtenstein GR: Tumor necrosis factor-alpha inhibitor therapy and fetal risk: A systematic literature review. World J Gastroenterol 19: 2591-2602, 2013.

7. Laharie D, Bourreille A, Branche J, Allez M, Bouhnik Y, Filippi J, Zerbib F, Savoye G, Nachury M, Moreau J, et al: Ciclosporin versus infliximab in patients with severe ulcerative colitis refractory to intravenous steroids: A parallel, open-label randomised controlled trial. Lancet 380: 1909-1915, 2012.

8. Singh S, Heien HC, Sangaralingham LR, Schilz SR, Kappelman MD, Shah ND, and Loftus EV: Comparative effectiveness and safety of infliximab and adalimumab in patients with ulcerative colitis. Aliment Pharmacol Ther 43: 994-1003, 2016.

9. Marehbian J, Arrighi HM, Hass S, Tian H and Sandborn WJ: Adverse events associated with common therapy regimens for moderate-to-severe Crohn's disease. Am J Gastroenterol 104: 2524-2533, 2009.

10. No authors listed: Can super-antibody drugs be tamed? Nature 440: 855-856, 2006.

11. Rolinski J and Hus I: Breaking immunotolerance of tumors: A new perspective for dendritic cell therapy. J Immunotoxicol 11: 311-318, 2014.

12. Park KB, Lim BK, Ye MB, Chung SY and Nam JH: A peptide vaccine based on a B-cell epitope on the VP1 protein of enterovirus 70 induces a strong antibody response. Acta Virol 56: 337-342, 2012.

13. Paul S and Piontkivska H: Frequent associations between CTL and T-Helper epitopes in HIV-1 genomes and implications for multi-epitope vaccine designs. BMC Microbiol 10: 212, 2010.

14. Haro I and Gómara MJ: Design of synthetic peptidic constructs for the vaccine development against viral infections. Curr Protein Pept Sci 5: 425-433, 2004.

15. Zhang J, Yang J, Fan D, Tao H, Wang H and Yu T: Peptide FLNPDVLDI of heparanase is a novel HLA-A2-restricted CTL epitope and elicits potent immunological antitumor effects in vitro with an 8-branched design. Oncol Rep 29: 1955-1961, 2013.

16. Zhang J, Yang J, Cai Y, Jin N, Wang H and Yu T: Multiple antigenic polypeptide composed of heparanase Bcell epitopes shrinks human hepatocellular carcinoma in mice. Oncol Rep 33: 1248-1256, 2015. 
17. Jia JY, Zhou HZ and Tang J: The study of mouse TNF- $\alpha$ functional domain and its neutralizing antibody binding site. Prog Biochem Biophys 36: 424-430, 2009 (In Chinese).

18. Boraschi D and Tagliabue A: Interleukin-1 and interleukin-1 fragments as vaccine adjuvants. Methods 19: 108-113, 1999.

19. Chakraborty S, Chakravorty R, Ahmed M, Rahman A, Waise TM Hassan F, Rahman M and Shamsuzzaman S: A computational approach for identification of epitopes in dengue virus envelope protein: A step towards designing a universal dengue vaccine targeting endemic regions. In Silico Biol 10: 235-246, 2010

20. Yang XS, Wang HQ, Yuan QF, Xie Y, Yao ZB, Ye XZ, Wu JM and Zhou AG: Directly synthesize A $\beta$ _(1-15) peptide vaccine by fmoc solid-phase peptide synthesis and study its immune activity. J Sun Yat Sen Uni 27: 121-125, 2006 (In Chinese).

21. Wang J, Sun N, Zhou C, Zhou X, Lu J, Wang C and Che H: Food proteins from different allergen families sensitize balb/c mice to family-specific immune responses. J Immunotoxicol 11: 172-179, 2014.

22. Zhang J, Cui Y, Wu Y, Wang $\mathrm{H}$ and Ke J: Prediction and identification of Bcell epitopes for tumor necrosis factoralpha. Mol Med Rep 16: 3439-3444, 2017.

23. Murano M, Maemura K, Hirata I, Toshina K, Nishikawa T, Hamamoto N, Sasaki S, Saitoh O and Katsu K: Therapeutic effect of intracolonically administered nuclear factor kappa B (p65) antisense oligonucleotide on mouse dextran sulphate sodium (DSS)-induced colitis. Clin Exp Immunol 120: 51-58, 2000.

24. Kihara N, de la Fuente SG, Fujino K, Takahashi T, Pappas TN and Mantyh CR: Vanilloid receptor-1 containing primary sensory neurones mediate dextran sulphate sodium induced colitis in rats. Gut 52: 713-719, 2003.

25. Sureshbabu A, Syed MA, Boddupalli CS, Dhodapkar MV, Homer RJ, Minoo P and Bhandari V: Conditional overexpression of TGF $\beta 1$ promotes pulmonary inflammation, apoptosis and mortality via TGF $\beta R 2$ in the developing mouse lung. Respir Res 16: 4, 2015.

26. Thorlund K, Druyts E, Mills EJ, Fedorak RN and Marshall JK Adalimumab versus infliximab for the treatment of moderate to severe ulcerative colitis in adult patients naive to anti-TNF therapy: An indirect treatment comparison meta-analysis. J Crohns Colitis 8: 571-581, 2014.

27. Fausel R and Afzali A: Biologics in the management of ulcerative colitis-comparative safety and efficacy of TNF-alpha antagonists. Ther Clin Risk Manag 11: 63-73, 2015.

28. Ben-Horin S, Kopylov U and Chowers Y: Optimizing anti-TNF treatments in inflammatory bowel disease. Autoimmun Rev 13 24-30, 2014.
29. de Mattos BR, Garcia MP, Nogueira JB, Paiatto LN, Albuquerque CG, Souza CL, Fernandes LG, Tamashiro WM and Simioni PU: Inflammatory bowel disease: An overview of immune mechanisms and biological treatments. Mediators Inflamm 2015: 493012, 2015.

30. Chey WY: Infliximab for patients with refractory ulcerative colitis. Inflamm Bowel Dis 7 (Suppl 1): S30-S33, 2001.

31. Okamoto R and Watanabe M: Functional relevance of intestinal epithelial cells in inflammatory bowel disease. Nihon Rinsho Meneki Gakkai Kaishi 39: 522-527, 2016.

32. Landy J, Ronde E, English N, Clark SK, Hart AL, Knight SC, Ciclitira PJ and Al-Hassi HO: Tight junctions in inflammatory bowel diseases and inflammatory bowel disease associated colorectal cancer. World J Gastroenterol 22: 3117-3126, 2016.

33. Noth R, Stüber E, Häsler R, Nikolaus S, Kühbacher T, Hampe J, Bewig B, Schreiber S and Arlt A: Anti-TNF- $\alpha$ antibodies improve intestinal barrier function in Crohn's disease. J Crohns Colitis 6: 464-469, 2012.

34. Di Sabatino A, Pender SL, Jackson CL, Prothero JD, Gordon JN, Picariello L, Rovedatti L, Docena G, Monteleone G, Rampton DS, et al: Functional modulation of Crohn's disease myofibroblasts by anti-tumor necrosis factor antibodies. Gastroenterology 133: 137-149, 2007.

35. Li JY, Lu Y and Fu XB: The significance of changes in diamine oxidase activity in intestinal injury after trauma. Chin Crit Care Med 12: 482-484, 2000 (In Chinese).

36. Leaphart CL and Tepas JJ III: The gut is a motor of organ system dysfunction. Surgery 141: 563-569, 2007.

37. Ivanov AI, Nusrat A and Parkos CA: Endocytosis of the apical junctional complex: Mechanisms and possible roles in regulation of epithelial barriers. Bioessays 27: 356-365, 2005.

38. Furuse M, Hirase T, Itoh M, Nagafuchi A, Yonemura $S$ and Tsukita S: Occludin: A novel integral membrane protein localizing at tight junctions. J Cell Biol 123: 1777-1788, 1993.

39. Wardill HR, Bowen JM, Al-Dasooqi N, Sultani M, Bateman E, Stansborough R, Shirren J and Gibson RJ: Irinotecan disrupts tight junction proteins within the gut: Implications for chemotherapy-induced gut toxicity. Cancer Biol Ther 15: 236-244, 2014

40. Shang HX, Wang AQ, Bao CH, Wu HG, Chen WF, Wu LY, Ji R, Zhao JM and Shi Y: Moxibustion combined with acupuncture increases tight junction protein expression in Crohn's disease patients. World J Gastroenterol 21: 4986-4996, 2015. 